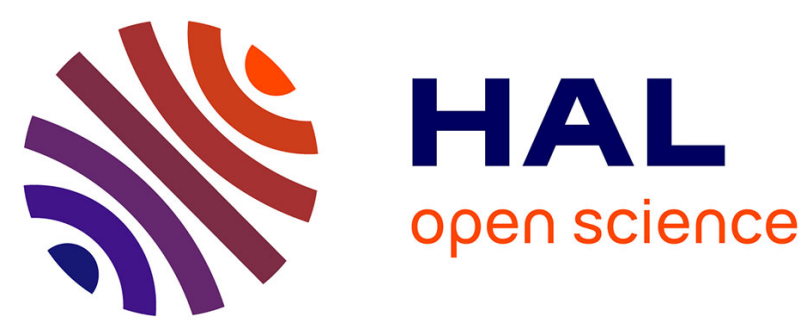

\title{
Analysis of degenerated aortic valve bioprosthesis by segmentation of preoperative CT images
}

Vito-Giovanni. Ruggieri, Wang Qian, Simon Esneault, Raphael Madeleine, Limin Luo, Alain Leguerrier, Jean-Philippe Verhoye, Pascal Haigron

\section{To cite this version:}

Vito-Giovanni. Ruggieri, Wang Qian, Simon Esneault, Raphael Madeleine, Limin Luo, et al.. Analysis of degenerated aortic valve bioprosthesis by segmentation of preoperative CT images. Innovation and Research in BioMedical engineering, 2012, 33 (5-6), pp.287-297. 10.1016/j.irbm.2012.09.001 . inserm00785803

\section{HAL Id: inserm-00785803 https://www.hal.inserm.fr/inserm-00785803}

Submitted on 7 Feb 2013

HAL is a multi-disciplinary open access archive for the deposit and dissemination of scientific research documents, whether they are published or not. The documents may come from teaching and research institutions in France or abroad, or from public or private research centers.
L'archive ouverte pluridisciplinaire HAL, est destinée au dépôt et à la diffusion de documents scientifiques de niveau recherche, publiés ou non, émanant des établissements d'enseignement et de recherche français ou étrangers, des laboratoires publics ou privés. 


\title{
Analysis of degenerated aortic valve bioprosthesis by segmentation of preoperative CT images
}

\section{Analyse de bioprothèse valvulaire aortique dégénérée par segmentation d'images TDM préopératoires}

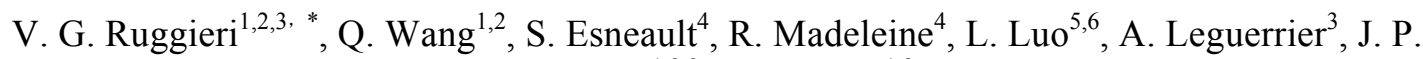 \\ Verhoye $^{1,2,3}$, P. Haigron ${ }^{1,2}$ \\ ${ }^{1}$ LTSI, université de Rennes 1, campus de Beaulieu, bâtiment 22, 35000 Rennes, France \\ ${ }^{2}$ Inserm U1099, campus de Beaulieu, bâtiment 22, 35000 Rennes, France \\ ${ }^{3}$ Department of Thoracic and Cardiovascular Surgery, University Hospital Pontchaillou, 35000 Rennes, France \\ ${ }^{4}$ Therenva, CIC-IT, Rennes, France \\ ${ }^{5}$ LIST, Southeast University, Nanjing, China \\ ${ }^{6}$ CRIBs, Centre de Recherche en Information Biomedicale sino-francais, Rennes / Nanjing, France / China.
}

\begin{abstract}
In the next future, transcatheter aortic valve implantation could represent a minimally invasive option in case of bioprosthesis failure for patients at high surgical risk. CT based preoperative planning of this procedure could be useful to optimize valve-in-valve implantation. In this context, bioprosthesis 3D analysis seems to be necessary, particularly for leaflets.

The goal of this study was to propose different methods to segment and characterize a degenerated bioprosthesis using standard preoperative CT scan images in order to map structural injury of bioprosthesis and, ultimately, to plan the best positioning for valve-in-valve implantation. We report our preliminary results on segmentation of a degenerated bioprosthesis in aortic position. Three different methods have been tested and all allowed obtaining segmentation of the different bioprosthesis components. Results were compared by means of quantitative criteria. Explanted bioprosthesis CT images were used as reference. Semi-automatic segmentation seems to provide an interesting approach for the morphological characterization of degenerated bioprosthesis.

\section{Résumé}

Dans le future prochain, l'implantation percutanée de prothèse valvulaire aortique pourrait représenter une option mini-invasive dans le cadre de la dégénérescence de bioprothèse chez les sujets à haut risque chirurgical. La planification préopératoire basée sur les images scanner pourrait être utile pour optimiser l'implantation de la valve dans la valve. Dans ce contexte, une analyse tridimensionnelle de la bioprothèse dégénérée parait nécessaire, surtout en ce qui concerne les feuillets prothétiques.

Le but de l'étude est de proposer différentes méthodes pour segmenter et caractériser une bioprothèse dégénérée en utilisant les images scanner préopératoires à fin de mapper l'endommagement structurel de la bioprothèse et, en dernier hypothèse (dans une optique future), de planifier le positionnement adapté de la valve percutanée dans la bioprothèse. Nous reportons les résultats préliminaires de la segmentation de bioprothèse valvulaire aortique. Trois différentes méthodes ont été testées et toutes ont permis d'obtenir une segmentation des différentes composantes de la bioprothèse. Les résultats ont été comparés par des critères quantitatifs. Les images scanner des bioprothèses explantées lors du ré-intervention ont servi de référence. La segmentation semi-automatique représente une approche intéressante pour la caractérisation morphologique des bioprothèses dégénérées.
\end{abstract}

Keywords: Aortic valve bioprosthesis analysis, valve-in-valve, stick based segmentation, aortic valve implantation

Mots-clés: Analyse de bioprothèse de valve aortique, valve dans valve, segmentation basée "stick", implantation de valve aortique.

*Auteur correspondent

Adresse e-mail : vito-giovanni.ruggieri@chu-rennes.fr (V.G. Ruggieri) 


\section{INTRODUCTION}

Aortic valve stenosis remains the most common valvular disease $[1,2]$. The gold standard for treatment is surgical aortic valve replacement [3]. The combined effects of an aging population and the prevalence of aortic stenosis in this cohort have led to increasing numbers of elderly patients being referred for valvular surgery. The valve of choice in these patients is usually a bioprosthetic device, to avoid the need for anticoagulation with its associated complications [4]. Moreover, with the good long term results in durability of the new generation devices, bioprosthesis implantation is going to increase also in younger patients in the next years.

As a result, there is an increasing prevalence of failing bioprosthetic aortic valve replacements. Concurrently, the spectrum of comorbidities in these patients grows more complex, and therefore the risks of re-do surgery increase. Absolute surgical risk for re-do aortic valve operations might be raised by up to $15 \%$ compared with initial implants $[5,6]$.

Trans-catheter aortic valve implantation (TAVI) has entered the mainstream as a viable treatment option for patients with symptomatic, severe aortic stenosis, who are at prohibitively high surgical risk. The initial success in 2002 [7] has been followed by a surge of interest, strengthened by encouraging medium-term results with both the self-expanding CoreValve (Medtronic, Luxembourg) [8,9] and balloon-expandable Edwards Sapien (Edwards Lifesciences, Irvine, California) systems [10,11].

Although not initially designed for this purpose, TAVI is also an option for patients with a degenerative aortic valve bioprosthesis who would be at high surgical risk from repeat thoracotomy. The feasability of implanting a trans-catheter valve into a surgical bioprosthesis was first demonstrated in a pig model in 2007 [12]. The investigators used the Cribier-Edwards system to transapically implant 23-mm devices into 5 Carpentier Edwards porcine aortic valve prostheses to good effect. A case report of successful treatment of a severely regurgitant aortic valve bioprosthesis with the CoreValve (Medtronic) system has also been described-with retention of good function and no complications at 1-year follow-up [13].

Until now, some reports about valve-in-valve implantation are available [14], but the implanting technique remains empiric and modified for each different case and prosthesis. In next future, preoperative patient-specific simulation of endovascular procedure could be useful to optimize valve-in-valve implantation in order to predict intra-operative fitting and to reach the best positioning of the new prosthesis. In order to realize a preoperative planning of valve-in-valve implantation, $3 \mathrm{D}$ analysis and characterization of the structures of interest are necessary.

Nowadays, automatic processing segmentation of preoperative vascular $\mathrm{CT}$ images is feasible and provides relatively accurate results. This is no more the case for valvular prosthesis, specifically for prosthetic leaflets and their degeneration. To our knowledge there are some methods reported in the literature dealing with coronary ostia and valve leaflets detection $[15,16]$. None of them deals with the issue of CT analysis of failing aortic bioprosthesis. The difficulties come from the combination of different factor: inhomogeneities of contrast product distribution used for the acquisition of CT images of cardiovascular structures, low contrast between the structures of interest (leaflets, calcifications, contrast) and image resolution compared with structure thickness (leaflets).

The goal of this study is to propose a new segmentation-based approach to characterize a degenerated bioprosthesis. We show and discuss our preliminary results. Results of the preoperative segmentation could, ultimately, be useful to discuss about the valve-in-valve procedure indication and to plan the best positioning for trans-catheter implantation.

\section{ANALYSIS OF DEGENERATED VALVE}

\section{A. Degenerated valve leaflets analysis}

Multi Slice Computed Tomography (MSCT) shows a high spatial resolution and can also be used to acquire spatio-temporal data during the cardiac cycle. In this work we are interested in using MSCT data to assess pathological features of cardiovascular structures such as aortic valve leaflets. Figure 1 illustrates original noisy MSCT images showing stent with high density and possible artifacts (A), low contrasted leaflets (B), calcifications on leaflets (C) and pannus under stent (D). The range of grey level in vessel lumen was typically about [1300 HU, $1550 \mathrm{HU}]$, and in leaflets about [1150 HU, $1420 \mathrm{HU}]$.

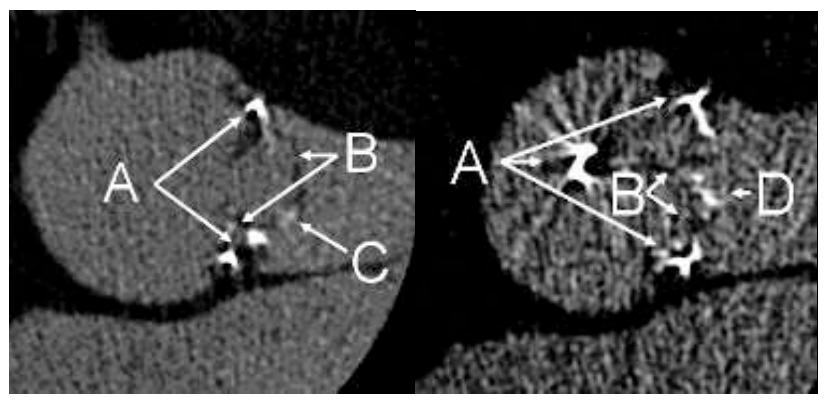

Figure1: Two noisy MSCT slices of aortic valve prosthesis showing stent (A), leaflets (B), calcification (C) and pannus (D).

The overall framework (Fig.2) proposed to analyze thin cardiovascular structures as valve leaflets from MSCT data, is composed of : (i) the definition of a Region of Interest (ROI) depending on the type of bioprosthesis stent implanted in the patient, (ii) a preprocessing step to denoise the original image, (iii) the segmentation of valve bioprosthesis components mainly based on a region growing process and (iv) the visualization process. Two approaches were considered for the preprocessing and three for the segmentation. In the preprocessing step anisotropic Diffusion curvature filter and Stick filter were implemented. In the segmentation process, the first approach was based on a classical isotropic region growing approach, the second on a directional (stick) region growing process and the third on a stick exhaustive search. In order to evaluate the performance of the segmentation of "in-vivo" images (preoperative images), the proposed approach was also applied to the "ex-vivo" images (explanted bioprosthesis) after patient reoperation. 


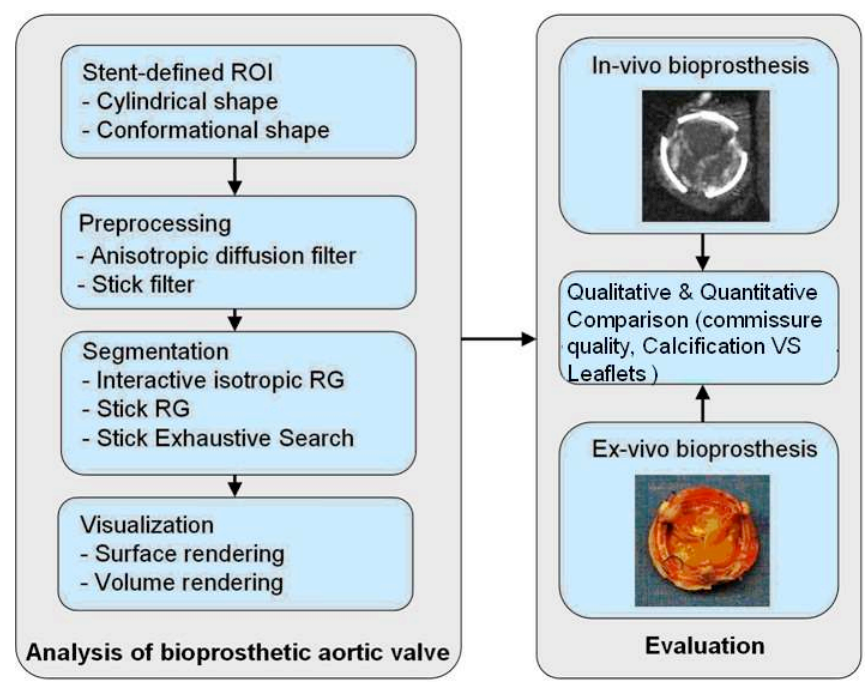

Figure 2: Framework of bioprosthetic aortic valve analysis from MSCT data.

\section{B. Stent defined ROI}

In order to deal with the difficulties of segmentation of the $\mathrm{CT}$ images of the bioprosthesis mentioned above (metal artifacts, thickness of leaflets compared with image resolution, density of valve components compared to stent) we considered a region of interest (ROI) defined by the stent. This ROI can have different shapes according to the type of stent implanted in the patient. We considered two kinds of shape for the ROI: a cylindrical shape preferentially for incomplete metallic stents and a conformational shape for complete metallic stents (Fig. 3).
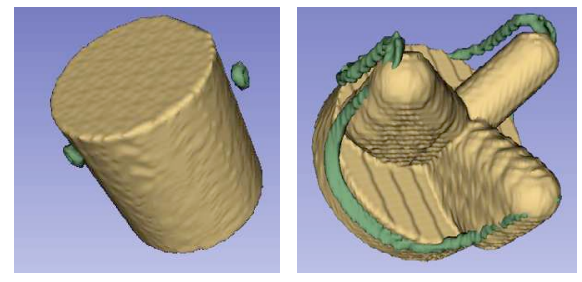

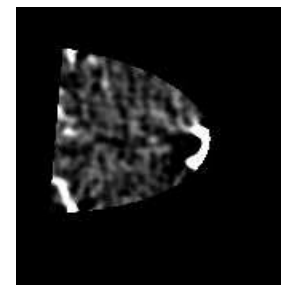

A

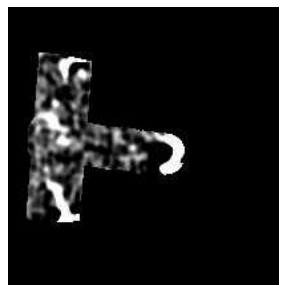

B
Figure 3: Shapes (A cylindrical, and B conformational) of stent defined ROI and illustration of the corresponding $\mathrm{CT}$ observations (within one of the original CT slices).

\section{Preprocessing}

In order to reduce the noise in CT images a preprocessing step is implemented. The preprocessing goal is to increase the signal to noise ratio (SNR) as well as to increase the contrast of the structures of interest, especially the valve leaflets. We considered methods based on Diffusion filters (curvature and anisotropic Diffusion filter) as well as an adaptation of Stick filters to reduce noise and keep details in thin structures. To select the most appropriate approach before the segmentation step, we considered the SNR as the performance criterion. To compute the SNR we used the definition given in [19]:

$$
S N R=\left.10 * \log _{10}\left(\frac{\operatorname{Max}\left(\operatorname{Var}_{i}\right)}{\operatorname{Min}\left(\operatorname{Var}_{i}\right)}\right)\right|_{i \in R O I},
$$

where $\operatorname{Var}_{i}$ is the the local variance in the neighborhood of leaflets and vascular lumen voxels located in the ROI. Voxels located at the boundary between leaflets and vascular lumen are used to obtain the maximum variance whereas voxels located in the vascular lumen are used to obtain minimum variance. Due to their high value, voxels representing stent, calcification and pannus are not considered in the computation of SNR.

\section{1 - Anisotropic Diffusion filter}

The Diffusion filters are non-linear filters based on partial differential equations. They have been proposed for noise reduction and segmentation, particularly for vascular images.

In classical Perona-Malik (PM) model [20], the values of resulting voxels $I_{p}^{t+1}$ are calculated as:

$$
I_{p}^{t+1}=I_{p}^{t}+\frac{\lambda}{\left|\eta_{p}\right|} \sum_{q \in \eta_{p}} c\left(\nabla I_{p, q}^{t}\right) \nabla I_{p, q}^{t},
$$

where $\eta_{p}$ is the neighborhood of the current voxel and $\left|\eta_{p}\right|$ is the number of voxels in $\eta_{p} . I_{p}^{t}$ is the current value of the voxel. $\nabla I_{p, q}^{t}$ is the discrete gradient along the $\mathrm{q}^{\text {th }}$ direction (defined by the current voxel and a voxel of the neighborhood). $c(x)$ is the classifier between edge and noise. $c(x)$ can take different expressions such as:

$$
c(x)=\frac{1}{1+(x / k)^{2}} \text { or } c(x)=\exp \left(-(x / k)^{2}\right),
$$

$\lambda$ and $k$ are two parameters to control Diffusion intensity.

$k=\left.\frac{1}{0.6745} \operatorname{median}\left(\left|\nabla I_{i}\right|-\right.$ median $\left.\left(\nabla I_{i}\right)\right)\right|_{I \in R O I}$ represents the absolute median value [21] of discrete gradient of all voxels inside ROI.

Classical Anisotropic Diffusion operators such as PM model can enhance edge while denoising the image. Nevertheless, they may enhance both edge and noise in low SNR conditions. Anisotropic Curvature Diffusion, which is based on a Modified Curvature Diffusion Equation [22-24], has been introduced to overcome this problem and to be less sensitive to noise.

Discrete Gradient $\nabla I_{p, q}^{t}$ used in PM model was replaced by Gaussian Curvature of the voxel in the Anisotropic Curvature Diffusion:

$$
I_{p}^{t+1}=I_{p}^{t}-\frac{\lambda}{N} \sum_{q \in \eta_{p}} c\left(\operatorname{Curv}\left(P_{p, q}^{t}\right)\right) \operatorname{Curv}\left(P_{p, q}^{t}\right),
$$


where $P_{p, q}^{t}$ is the plane defined by the current voxel and a direction given by a voxel of the neighborhood $\eta_{p}$. $\operatorname{Curv}\left(P_{p, q}^{t}\right)$ is the Gaussian Curvature at the voxel. $N=3 L^{2}-6 L+4, \quad \mathrm{~L}$ is the length of side of cubic neighborhood.

In the proposed $\mathrm{CT}$ data preprocessing approach, we chose $\left|\eta_{p}\right|=26$ and $\mathrm{N}=13 \quad(3 * 3 * 3$ cubic neighborhood).

Higher values of $\eta_{p}$ may smooth edge and increase computational complexity too much. $c(x)=\frac{1}{1+(x / k)^{2}}$ was selected thanks to its simple computational complexity. Values of $\lambda$ and $\mathrm{k}$ were set at 1 for a low level of noise (high SNR). In the case of images showing a high level of noise $(\mathrm{SNR}<=8), \lambda$ were set at 0.5 .

\section{2 - Stick filter}

Stick filter is also a non-linear filter. It was originally introduced to deal with strips-like noise in US images [17-18]. With Stick filtering approach, the neighborhood of the considered voxel is divided into a set of asymmetric sticks to perform a non-linear filtering of the image (Fig. 4). Because of its asymmetric stick, the filter could get better performance in terms of keeping details of thin structure, as valve leaflets, while denoising the image.

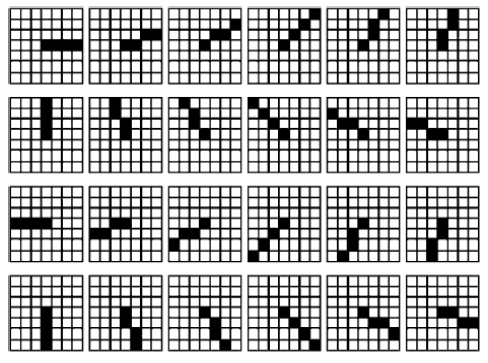

A

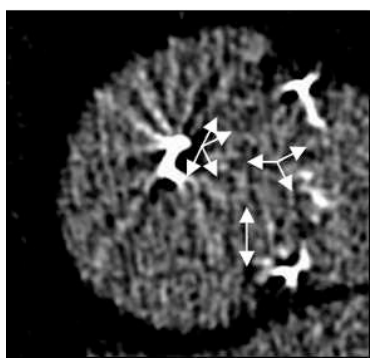

B
Figure $4:$ A, 4 voxels-length $2 \mathrm{D}$ asymmetric sticks set [18] and B, 5 voxel-length sticks (white arrows) which give the higher output in 4 positions inside leaflets (SNR of this image is 7.54).

The filter output I' at the current voxel was originally defined as:

$$
I^{\prime}=\frac{1}{W} \sum_{i=1}^{N} g_{i} \bullet \bar{I}_{i}, \text { and } W=\sum_{i=1}^{N} g_{i}
$$

where $\bar{I}_{i}$ denotes the local mean value along the $i^{\text {th }}$ stick, $N$ is the number of sticks, and $g_{i}$ is the reciprocal of local variance along the $i^{\text {th }}$ stick, used to classify edge and noise.

This filter was adapted to preprocess CT images of degenerative aortic valve bioprosthesis. In order to improve the results of the original stick filter applied to CT images, we tested different parameters (including local median). At the end of the tests, we found that local median could keep more details of thin structures both visually and in terms of SNR.
In our approach, we used 3D asymmetric sticks set and the resulting value of the filtering process was considered as the sum of voxel median values normalized by the standard deviation along 4 voxels length sticks defined in the neighborhood (Fig. 5). The values of resulting voxels $I^{\prime}$ were more precisely defined as:

$$
I^{\prime}=\frac{1}{W} \sum_{i=1}^{N} g_{i} \bullet I_{M i}, \text { and } W=\sum_{i=1}^{N} g_{i}
$$

where $I_{M i}$ denotes the local median value along the $i^{\text {th }}$ stick, $N$ is the number of sticks $\left(N=24 L^{2}-48 L+26\right.$ in $3 \mathrm{D}$ conditions), $L$ is the number of voxels along the stick. $L$, the length of stick, is the main parameter of this filter. It is determined by SNR of original CT images. When $\mathrm{SNR}<=8$, $L=5 ; 8<\mathrm{SNR}<=10, L=4$; $\mathrm{SNR}>10, L=3$.

The process of filtering is applied to the ROI. When part of stick is outside of the ROI, we use original image voxels to complete it.

Besides, the number of iteration is not a critical issue unlike Diffusion filter. Stick filter shows a convergent behavior after several iterations. Typically, in a low SNR condition $(\mathrm{SNR}<=8)$, the result converged after 5 iterations (after 3 iterations when $\mathrm{SNR}>10$ ). It was not necessary to limit the number of iterations to avoid an over-smoothing of edges.

Although both Stick filter and Diffusion filter are "anisotropic" filter, Diffusion often use a small mask $(3 * 3 * 3)$ or add a median filter process between 2 iterations to avoid making impulse noise. Stick filter can use much larger mask to get a better result.

The drawback of Stick filter is its high computation complexity. A 5 voxels-length Stick filter (i.e. a 9*9*9 cubic neighborhood), requires for each voxel and at each iteration, the calculation of 386 local medians and local variances. The time of calculation can be decreased by the stent-defined ROI. The number of voxels located in the ROI is much less than in the cubic volume encompassing the aortic valve bioprosthesis. Cylindrical ROI typically decreases the number of voxels to $40 \%$ and conformational ROI to $16 \%$.

\section{Valve segmentation}

According to surgical expertise, four main classes of tissues were identified. They were related to HU measured by CT. We considered, from high to low HU, the following classes: stent, calcification, vascular lumen and leaflets. The first two elements, showing high CT values, can be segmented by thresholding easily; the issue of segmentation is more particularly focused on bioprosthetic leaflets.

Three kinds of region-based segmentation processes were implemented, with a decreasing level of user interactivity.

\section{1- Isotropic Region Growing}

The first one was an interactive process in order to provide a reference approach for the assessment of the results. It was based on classical Isotropic Region Growing 
(IRG) method. Following a preprocessing based on Diffusion filtering, as described above, it was applied using 3D Slicer software [25].

The bioprosthesis volume was selected using three different CT slices. Multiple seed points were interactively selected for each component of the prosthesis. The region growing algorithm was started (26-neighbour connectivity) with automatic calculation of the criterion used for the aggregation of voxels. This similarity criterion was based on mean and standard deviation of the voxel values within the considered cubic neighborhood [26-28]. The extraction and visualization of the resulting 3D surface meshes delineating the regions were performed by using the Marching Cube algorithm $[19,25]$.

\section{2 - Stick Region Growing}

To better deal with the segmentation of thin structures, like the components of a valve bioprosthesis and especially valve leaflets, we developed a directional region growing process based on stick neighborhood. The segmentation was thus performed by the stick region growing algorithm applied to a previously selected region of interest (ROI), either cylindrical or conformational, passing through the metallic stent.

Some seeds were interactively selected inside the leaflets at different locations (typically 4 to 6 seeds). A set of $\mathrm{N}$ voxels inside the leaflets was defined by considering for each seed the voxels belonging to their neighborhood. The similarity criterion was locally based on mean and standard deviation of the CT voxel values along the directions defined by the sticks. This approach was implemented using MatLab development tools.

In order to examine each voxel in the cubic neighborhood of the seed, we specifically computed the stick variances in the set of sticks defined by each of these voxels:

$$
\operatorname{Var}=\frac{\sum_{j=1}^{L}\left(I_{i, j}-\bar{I}_{i}\right)^{2}}{L}, \text { where } I_{i, j} \text { is the grey level of } \mathrm{j}^{\text {th }} \text { voxel in }
$$

L was chosen to be less than the thickness of the leaflets. Typically $\mathrm{L}$ was between 3 and 5 voxels (about $1 \mathrm{~mm}$ ).

To ensure region growth is not early stopped, or in other words to limit the number of seed points selection in some critical cases (highly noisy data), the size of the cubic neighborhood was chosen to be large enough to include space between homogeneous fragment of leaflets. We typically used a 342-connected neighborhood ( $7 \times 7 \times 7)$.

We also defined $\overline{I_{m}}=\frac{1}{L} \sum_{j=1}^{L} I_{m, j}$, the local mean of the stick with minimum local variance.

The voxels were classified as belonging to the region of the seed voxel (i.e. as a voxel of normal leaflet tissue) when: (i) $\overline{I_{m}}$ belongs to the grey level range of normal leaflets and (ii) minimum local variance along stick is lower than a variance threshold (VTH). VTH was determined by the difference of local cubic neighborhood variances of leaflets voxels and boundary voxels. These variances have been previously calculated in SNR computation (for image preprocessing). When this voxel belongs to ROI, it constitutes new seed voxels. This process was iterated until no new voxel can be marked as a seed. This segmentation referenced as Stick Region Growing (Stick RG) in the following was preceded by a Stick filtering preprocessing step.

\section{3 - Stick Exhaustive Search}

To further reduce the level of interactivity in the segmentation process, a systematic exploration of the ROI was implemented with no need for seed point definition. This segmentation referenced as Stick Exhaustive Search (SES) in the following was preceded by a Stick filtering preprocessing step.

Looking at the grey level of leaflets/vascular lumen in the preselected and filtered ROI, we manually set the range of grey level thresholds about mean and variance. We divide neighborhood (Radius $=3$ pixels) of all voxels inside ROI into an asymmetric sticks sets and we automatically calculate local means and local variances along sticks. If the stick values were inside the range we set, the neighborhood voxel was classified as leaflets or vascular lumen. The exploration of the ROI is completely automatic.

\section{RESULTS}

\section{A. Experimental data}

As preoperative assessment, we obtained CT scan images (General ElectricTM Lightspeed ${ }^{\circledR}$ VCT 64) from four patients (HER, MAG, BOU, DAV) presenting a degeneration of aortic bioprosthesis. Depending on the case, the reoperation was performed from 7 to 19 years after implantation. Acquisition protocol was a multi-slice coronary angio-CT-scan ECG gated in 3D + T mode.

We analyzed 4 databases of aortic bioprostheses, explanted for different pathological reasons. In one case it was a pericardial bioprosthesis (HER), in which bovine pericardium is cut to reproduce aortic valve leaflets that are sewn on a complete metallic stent. Three more cases were represented by porcine bioprostheses, in which a porcine aortic valve is mounted on a complete metallic stent ( 2 cases: MAG and BOU) or incomplete metallic stent (1 case: DAV).

CT-scan sequences were preprocessed and segmented (as described in Fig. 2) to describe the vascular structure as well as the bioprosthesis metallic stent. After reoperation, CT-scan images of isolated explanted aortic bioprosthesis were obtained using the highest image resolution protocol. The main parameters derived from the CT acquisitions of in-vivo (IV) and ex-vivo (EV) aortic bioprosthesis, for each of the four cases, are summarized in Table I. 
TABLE I: MAIN PARAMETERS OF IN-VIVO (IV) AND EX-VIVO (EV) ACQUISITIONS

\begin{tabular}{|c|c|c|c|}
\hline Patient & Observation & Image size (voxels in $\mathrm{X}, \mathrm{Y}, \mathrm{Z}$ ) & Image sampling (mm in $\mathrm{X}, \mathrm{Y}, \mathrm{Z}$ ) \\
\hline \multirow[t]{2}{*}{ HER } & IV & 512512281 & $\begin{array}{llll}0.3125 & 0.3125 & 0.625\end{array}$ \\
\hline & EV & 512512198 & $0.3125 \quad 0.3125 \quad 0.3125$ \\
\hline \multirow[t]{2}{*}{ MAG } & IV & 512512105 & $\begin{array}{llll}0.318 & 0.318 & 0.312\end{array}$ \\
\hline & $\mathrm{EV}$ & 512512110 & $\begin{array}{llll}0.0966 & 0.0977 & 0.312\end{array}$ \\
\hline \multirow[t]{2}{*}{ BOU } & IV & 512512153 & 0.36320 .36320 .625 \\
\hline & $\mathrm{EV}$ & 512512120 & $\begin{array}{llll}0.0977 & 0.0977 & 0.312\end{array}$ \\
\hline \multirow[t]{2}{*}{ DAV } & IV & 512512290 & $0.418 \quad 0.418 \quad 0.635$ \\
\hline & $\mathrm{EV}$ & 512512125 & $\begin{array}{llll}0.0977 & 0.0977 & 0.3125\end{array}$ \\
\hline
\end{tabular}

\section{B. Preprocessing}

The preprocessing based either on Diffusion filter or on Stick filter was applied to the in-vivo CT volumes of the four patients. The bioprosthesis voxels were first isolated by considering the ROI defined by the stent. Ex-vivo images do not need preprocessing. Differences in grey level between stent, leaflets and calcification/pannus are very clear in ex-vivo data.

Examples of original images and resulting images obtained with the two different filters are reported in Figure 5. A first qualitative appreciation of the results let appears that Stick filter based approach seems to better enhance the contrast between valve leaflets and surrounding structures.

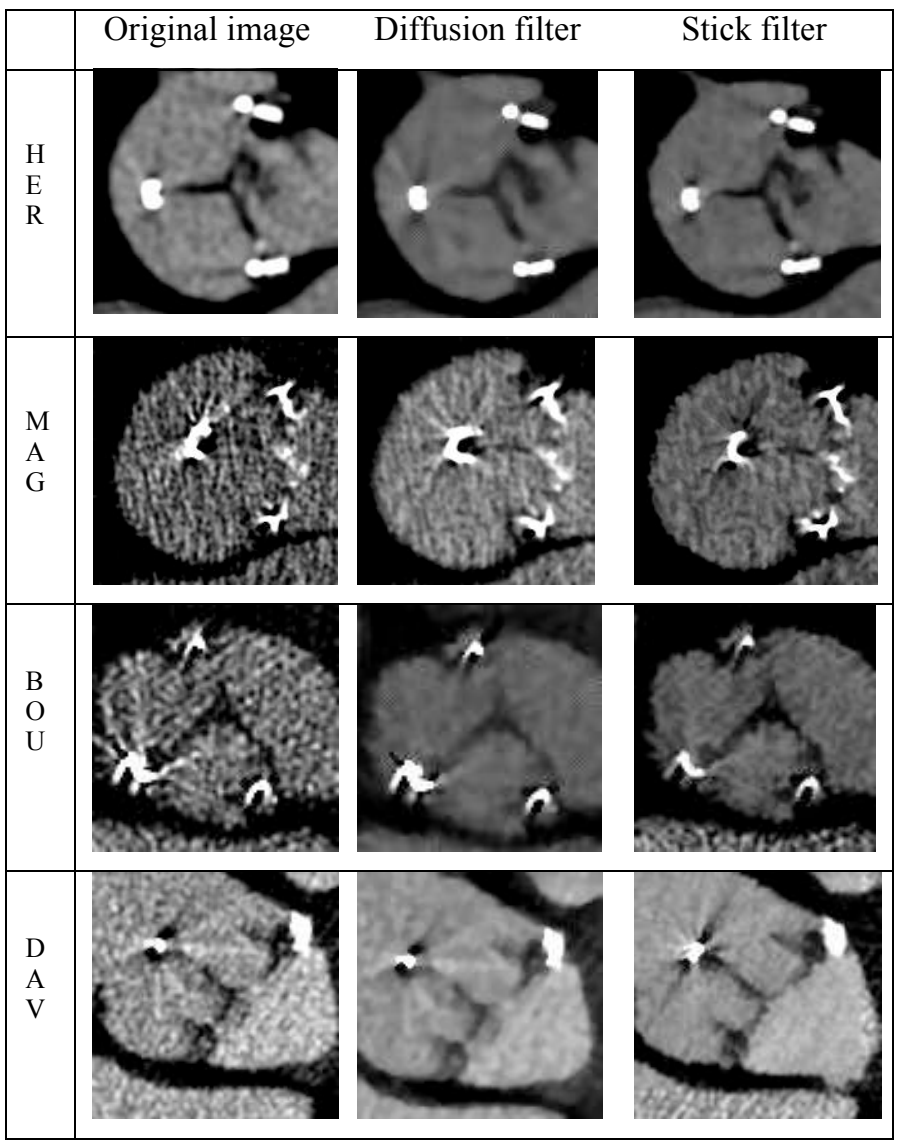

Figure 5: Results of CT images preprocessing by Diffusion and Stick filters.

In addition to this qualitative assessment of the results, SNR was computed on original images and resulting images
(Fig. 6). This objective assessment let appear that Stick filtering shows the best SNR for the four patients, and this is consistent with the subjective appreciation.

SNR

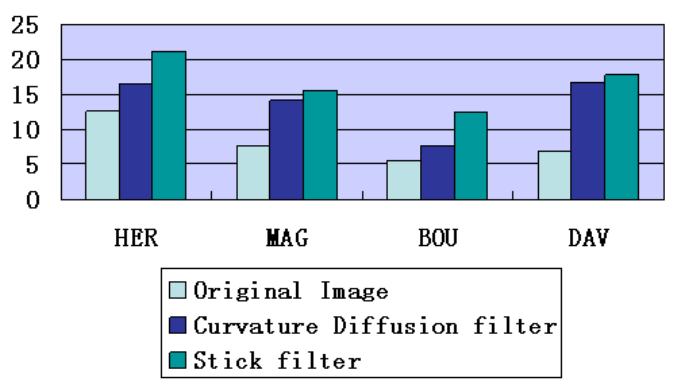

Figure 6: Results of preprocessing (SNR) with Stick filter and Curvature Diffusion filter for each of the four cases (HER, MAG, BOU and DAV).

\section{Segmentation}

Following the ad hoc preprocessing step, the different segmentation methods (IRG, Stick RG, and SES) were applied to in-vivo (IV) and ex-vivo (EV) images. A qualitative analysis is reported in Fig. 8-9 comparing the methods used in- and ex-vivo to enhance bioprosthetic leaflets and the explanted bioprosthesis.

The first example is reported in the case of the pericardial bioprosthesis (HER). The visualization was performed by surface rendering. Some anatomical similarity as the shape of the leaflets and the presence of minimal leaflet degeneration can be easily appreciated (Fig.7). The leaflet prolapse resulting in no perfect coaptation appears as commissure lost (indicated in blue circle) in the segmented images.
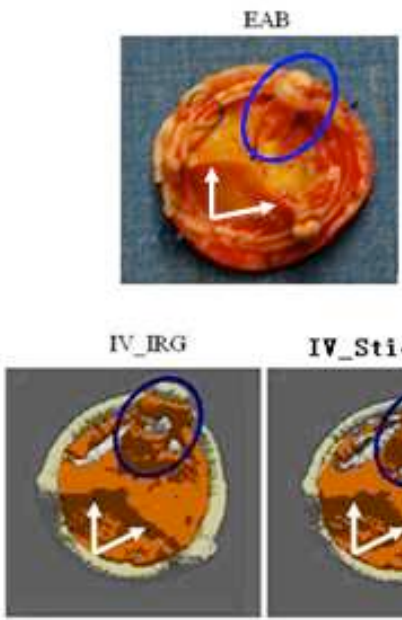

IV_Stick RG
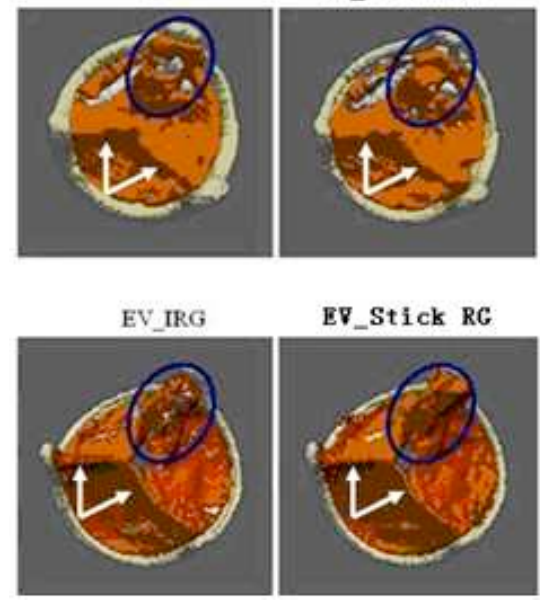

EV_Stick RG

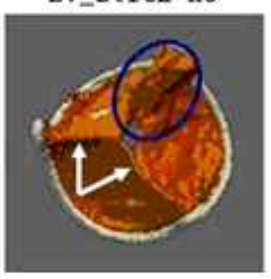


Figure 7: Visualization of the segmentation results obtained in case HER: explanted aortic bioprosthesis (EAB), in-vivo bioprosthesis (IV) and ex-vivo bioprosthesis (EV), segmented with methods IRG and Stick RG. The commissure lost is indicated with blue circle and good leaflets coaptation with white arrows.

The second example illustrates the results obtained in the case of a porcine bioprothesis (MAG). The visualization of front and back side of bioprosthesis was performed by volume rendering with transparency effect (Fig. 8). A calcified pannus (indicated by black arrows) is characteristic in this case and responsible for valve obstruction. Leaflet tear (indicated by green arrows) was also observed and found in reconstructions.

The results on the back side of EV_IRG and EV_SES are not exactly the same. In fact, in this case, when the valve is closed (closed position of leaflets), the pannus developed under the bioprosthesis is in contact with a part of the leaflets (partially calcified). Automatic enhancement of these 2 tissues in contact is quiet difficult. This is the reason why the results show some differences between the methods.

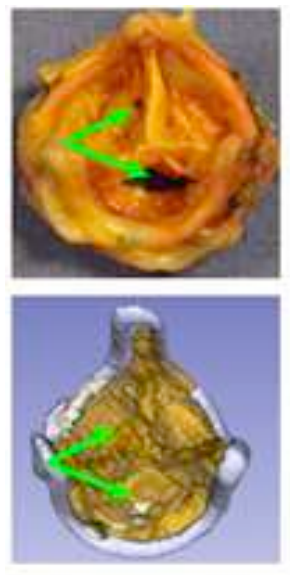

EAB

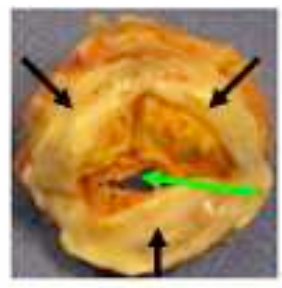

IV_IRG
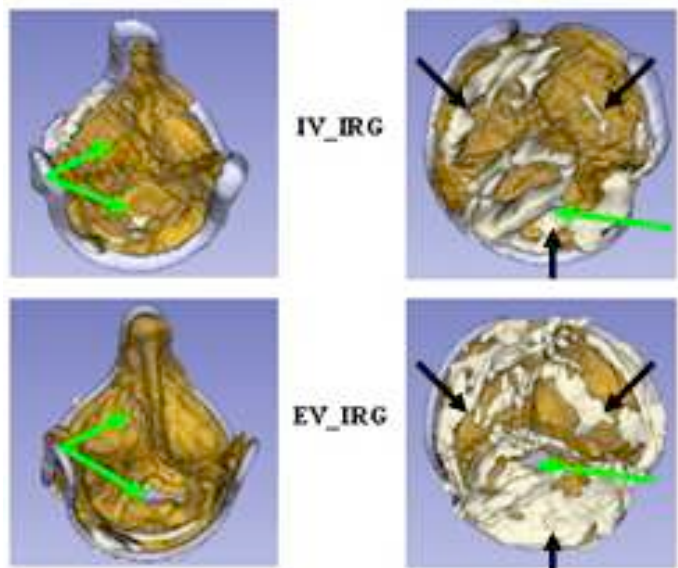

EV_IRG

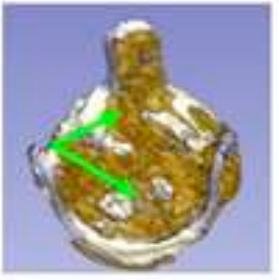

IV_SES

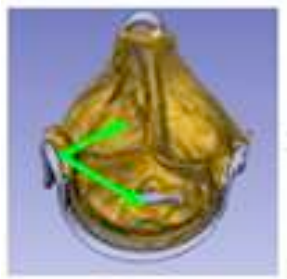

EV_SES
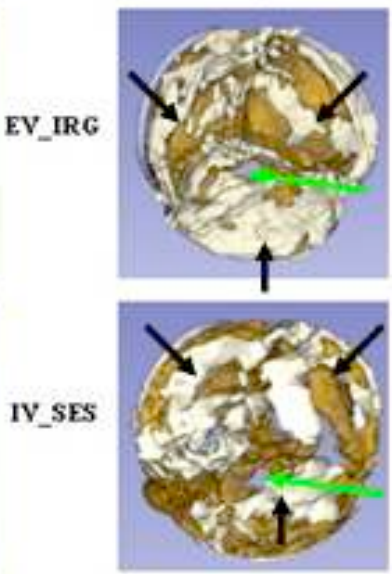

Front

Back
Figure 8: Visualization of the segmentation results obtained in case MAG (Front and Back side) explanted aortic bioprosthesis (EAB), in-vivo bioprosthesis (IV) and ex-vivo bioprosthesis (EV), segmented with methods IRG and SES. Calcified pannus (indicated by black arrows) and leaflet tears (indicated by green arrows) are observed and found.

A quantitative analysis was then conducted to objectively compare the results obtained respectively by IRG, Stick RG, and SES on in-vivo (IV) and ex-vivo (EV) images. It was performed as following: the voxel volume was computed for each database (considering CT acquisition parameters showed in Tab. I) and multiplied for the number of voxels of each bioprosthestic component.

The criterion used to evaluate the results was the volume of bioprothesis components. Results of this analysis are shown in Fig. 9 (volumes are expressed in $\mathrm{mm}^{3}$ ). This analysis wants to show that these 3 different methods more or less automatic, allow to enhance bioprosthetic components including leaflets with similar results. In fact, looking at volumes values, we can't conclude that the 3 methods provide so different results. In-vivo volumes are generally bigger than ex-vivo and it can be explained mostly by the moving artifacts. For sure, ex-vivo results are more reliable than in-vivo and represent the reference to compare the in-vivo results. But, when we compare the results between in- and ex-vivo for each method, the differences in voxels volumes are always less than $20 \%$.

\section{IV_Stent}

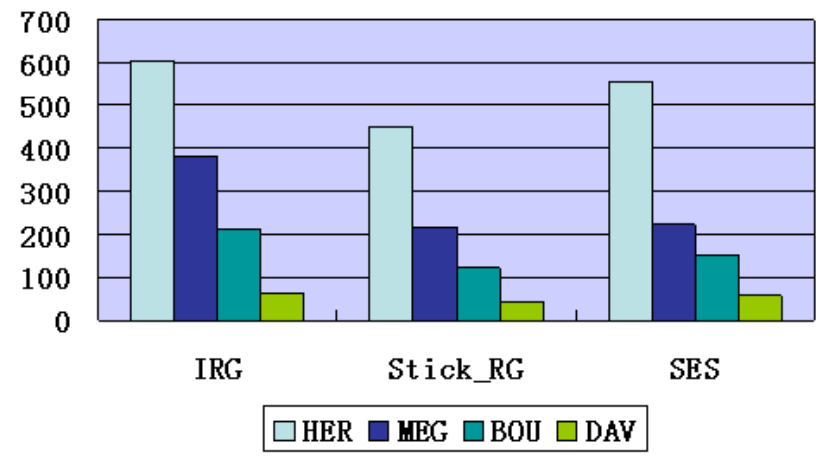

EV_Stent

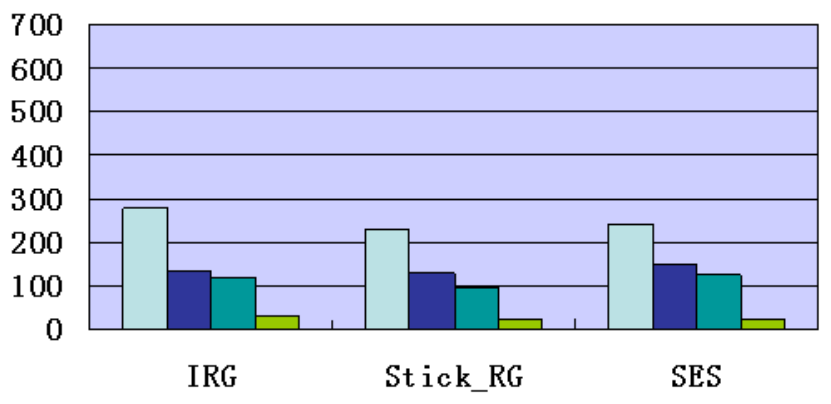

$\square$ HER $\square$ HAG $\square$ BOU $\square$ DAV 
IV_Leaflets

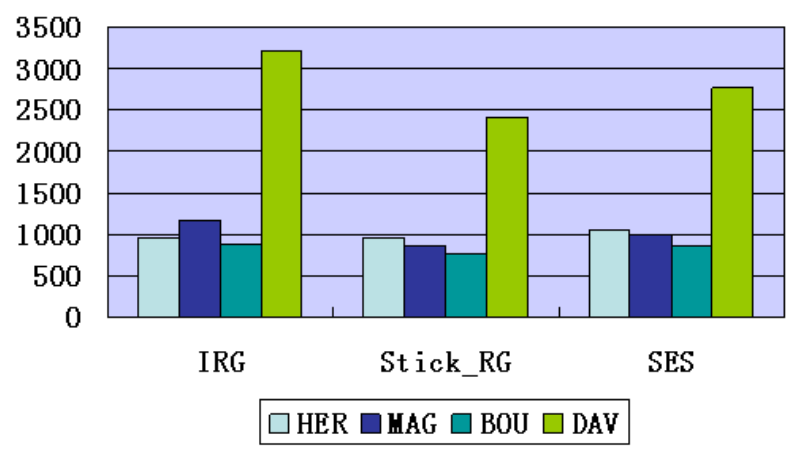

EV_Leaflets

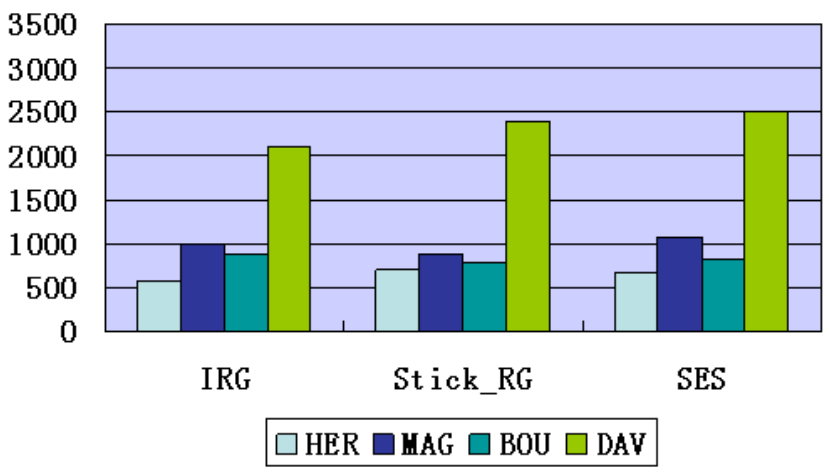

IV_Calcification/Pannus

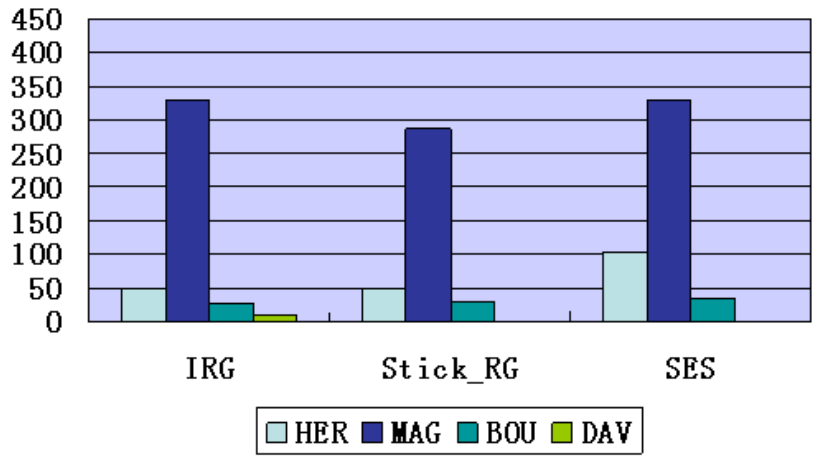

EV_Calcification/Pannus

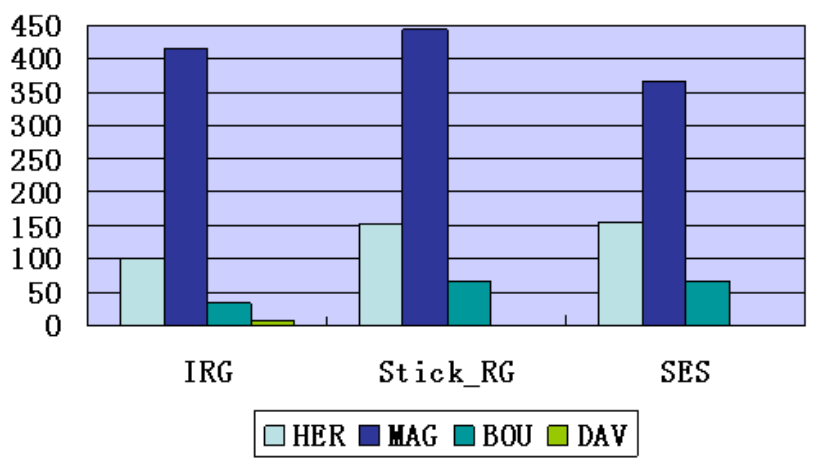

Figure 9: Volume analysis of bioprosthesis components

\section{DISCUSSION}

In recent years, multi-detector computed tomography has emerged as a promising tool to provide precise diagnostic information in different clinical situations, such as noninvasive evaluation of coronary disease [29-34], ventricular morphology and function [35], and myocardial viability [36-38]. CT images can also help visualize the morphology and motion of native and diseased stenotic aortic valves and precisely measure aortic valve opening areas. Several studies [39-45] suggest that multi-detector CT can help assess mechanism of dysfunction in mechanical prosthetic heart valve disorders.

An aortic bioprosthesis can have functional degradation and prosthesis dysfunction over time. In more recent bioprostheses, the rate of structural failure appears to be somewhat lower than with the first generation [46], but it is still occurring. Understanding the precise mechanism of bioprosthesis dysfunction would thus be important to direct clinical management. Yet, it can often be difficult to identify these entities in clinical practice. Indeed, both TTE and transesophageal echocardiography have limited usefulness for detection of the mechanism of stenotic bioprostheses, because of the acoustic shadowing from valve stents or annulus.

CT images would potentially allow better visualization of the bioprosthetic valve leaflets after aortic valve replacement, but at the moment CT scan softwares don't allow automatic leaflets enhancement. So, additional methods have to be considered to improve leaflets characterization and visualization.

In this study we have tested three different types of segmentation applied to CT images of degenerated aortic bioprosthesis. Considered methods, even if different in their approach, seem to be similar in terms of results. They are considered as semiautomatic segmentation methods.

The mean difference among these methods is that the Isotropic Region Growing method is performed using software for image analysis (3D Slicer), relatively easy to use, but needing heavy interaction for the user (manual selection of different seed points for the different components of the bioprosthesis). On the other hand, Stick Region Growing and Stick Exhaustive Search methods need, at this time, to be performed by professionals of Matlab development tools, but their interactivity is lower.

The reduced interactivity level results in some differences in terms of voxels volumes. In fact it is difficult to replace the user ability to recognize bioprosthesis different structures in CT transverse sections (IRG) by the automatic criteria based on grey level density (Stick RG and SES).

Quantitative analysis shows these differences among the methods, but we can't consider them significant because of the inability to perform a statistical analysis on these few databases. Moreover, we don't know at the moment if these small differences (always less than 20\%) could have a real interest for clinical application. In fact, the goal of segmentation is to approximately reconstruct leaflet morphology and to detect some failing mechanism as pannus 
formation that could represent a contraindication to the endovascular procedure.

A key point of the analysis is represented by the preprocessing. In fact, bioprostheses CT images need to be denoised before to be segmented because of metallic stent artifacts. Different filters have been tested in order to improve images with the best leaflets enhancement. Stick filtering, modified in order to be applied to CT images, showed the best visual results and this was confirmed by SNR analyses. This filtering process was performed by MatLab software and it takes time to be finalized. To reduce this computing time it is important to select a ROI that takes into account only the bioprosthesis stent region with its leaflets.

Concerning segmentations results, some considerations have to be done. Among analyzed databases the best results have been obtained in the case of the pericardial bioprosthesis (HER). The reason is attributed to the thickness of valve tissue. In fact pericardial leaflets are largely thicker than porcine with consequent better results for CT images.

The higher bioprosthesis components' volumes observed in some cases for in-vivo images comparing to ex-vivo can be explained by different factors. Firstly, image resolution is not the same. In-vivo acquisition protocol represents the standard protocol used for coronary ECG gated angio-CT-scan. Acquisition parameters cannot be changed and specific acquisition for bioprosthesis analysis doesn't exist at the moment. For ex-vivo we chose the highest resolution protocol to be close to reality and to use these images as reference. Other than image resolution, we can consider heart motion and blood superposition as important factor of volumes differences.

Computing of leaflets volumes can be altered by the non-metallic part of bioprosthesis stent (DAV). Only the metallic stent can be used as spatial limitation for the leaflets because the non metallic part has the same density of the leaflets. For this reason leaflet volumes are higher than normal, especially in the case of incomplete metallic stent.

Another factor that plays a fundamental role for the quality of in-vivo images (preoperative images) is the heart rate during the exam. It should be as low as possible and not more than $80 \mathrm{bpm}$. Patient preoperative clinical conditions can be responsible for higher heart rate and contra-indicate medical therapy as beta-blockers to reduce it.

In all cases, we observed also in-vivo underestimation of leaflets degeneration. This is only partially true. In fact, in the study cases, leaflets are not so calcified and most part of degeneration consists in leaflets thickening. These leaflets modifications do not appear with the same density of calcifications and are difficult to detect in in-vivo CT images. Only actual calcifications can be detected with consequent underestimation compared to ex-vivo images, in which smaller differences in density can be easily appreciated.

Our study suggests that segmentation of CT images could play a role in clarifying the mechanism of bioprosthesis dysfunction. Indeed, we demonstrated that segmentation can help directly visualize leaflet morphology.
Furthermore, we demonstrated the ability of these methods to identify bioprosthesis leaflet thickening, calcification as well as presence of pannus under bioprosthetic leaflets.

\section{CONCLUSION}

Echocardiography remains the gold standard for functional analysis of bioprosthesis failure. In the last years an increasing number of groups are looking with particular interest at CT images for anatomo-morphological analysis of the aortic valve.

Segmentation could be useful in the next future to better understand the morphology of the failing bioprosthesis. Moreover segmentation allows to better visualize some non structural valve dysfunction like subvalvular pannus, difficult to visualize by echocardiography and to recognize by transverse CT scan sections. Finally, it could be interesting to integrate $3 \mathrm{D}$ reconstructions into planning tools to optimize the valve-in-valve procedure.

In order to further validate the preliminary results, we are testing the three presented methods for different patients datasets.

Moreover additional types of segmentation approaches are going to be evaluated. Results among different techniques will be compared in order to determine the best method for a relevant $3 \mathrm{D}$ bioprosthesis segmentation.

\section{REFERENCES}

[1] Lindroos M, Kupari M, Heikkila J, Tilvis R. Prevalence of aortic valve abnormalities in the elderly: an echocardiographic study of a random population sample, J Am Coll Cardiol 1993;21:1220-1225.

[2] Iung B, Baron G, Butchart E G, Delahaye F, Gohlke-Barwolf C Levang $\mathrm{O} \mathrm{W}$, et al. A prospective survey of patients with valvular heart disease, in Europe: The Euro Heart Survey on Valvular Heart Disease. Eur Heart J 2003;24:1231-1243.

[3] Kojodjojo P, Gohil P, Barker D, Youssefi P, Salukhe T V, Choong A, et al. Outcomes of elderly patients aged 80 and over with symptomatic, severe aortic stenosis: impact of patient's choice of refusing aortic valve replacement on survival. QJM 2008;101:567-573.

[4] Accola KD, Scott ML, Palmer GJ, Thompson PA, Sand ME, Suarez-Cavalier JE, et al. Surgical management of aortic valve disease in the elderly: a retrospective comparative study of valve choice using propensity score analysis, J Heart Valve Dis 2008;17:355-364.

[5] Eitz T, Fritzsche D, Kleikamp G, Zittermann A, Horstkotte H, Korfer $\mathrm{R}$, et al. Reoperation of the aortic valve in octogenarians, Ann Thorac Surg 2006;82:1385-1390.

[6] Jamieson W R, Burr L H, Miyagishima R T. Re-operation for bioprosthetic aortic structural failure-risk assessment, Eur J Cardiothorac Surg 2003;24:873-878.

[7] Cribier A, Eltchaninoff H, Bash A, Borenstein N, Tron C, Bauer F, et al. Percutaneous transcatheter implantation of an aortic valve prosthesis for calcific aortic stenosis: first human case description, Circulation 2002;106:3006-3008.

[8] Grube E, Laborde J C, Gerckens U, et al. Percutaneous implantation of the CoreValve self-expanding valve prosthesis in high-risk patients with aortic valve disease: the Siegburg first-in-man study, Circulation 2006;114:1616-1624.

[9] Grube E, Schuler G, Buellesfeld L, Laborde J C, Gerckens U, Felderhoff $\mathrm{T}$, et al. Percutaneous aortic valve replacement for severe aortic stenosis in high-risk patients using the second- and current third-generation self-expanding CoreValve prosthesis: device success and 30-day clinical outcome, J Am Coll Cardiol 2007;50:69-76.

[10] Cribier A, H. Eltchaninoff, C. Tron, et al. Early experience with percutaneous transcatheter implantation of heart valve prosthesis for 
the treatment of end-stage inoperable patients with calcific aortic stenosis, J Am Coll Cardiol 2004;43:698-703.

[11] A. Cribier, Eltchaninoff H, Tron C, Bauer F, Agatiello C, Sebagh L, et al. Treatment of calcific aortic stenosis with the percutaneous heart valve: mid-term follow-up from the initial feasibility studies: the French experience, J Am Coll Cardiol 2006;47:1214-1223.

[12] Walther T, Falk V, Dewey T, Kempfert J, Emrich F, Pfannmuller B, et al. Valve-in-a-valve concept for transcatheter minimally invasive repeat xenograft implantation, J Am Coll Cardiol 2007;50:56-60.

[13] Wenaweser P, Buellesfeld L, Gerckens U, Grube E. Percutaneous aortic valve replacement for severe aortic regurgitation in degenerated bioprosthesis: the first valve in valve procedure using the Corevalve Revalving system, Catheter Cardiovasc Interv 2007;70:760-764.

[14] Webb J G, Wood D A, Gurvitch R Ye J, Masson J B, Rodes-Cabau J, et al. Transcatheter Valve-in-Valve Implantation for Failed Bioprosthetic Heart Valves, Circulation. 2010;121:1848-1857.

[15] Zheng Y, Matthias J, Rui L. Automatic Aorta Segmentation and Valve Landmark Detection in C-Arm CT: Application to Aortic Valve Implantation, MICCAI 2010, Part I, LNCS 6361, 2010: 476-483.

[16] Waechter I, Kneser R, Korosoglou G. Patient Specific Models for Planning and Guidance of Minimally Invasive Aortic Valve Implantation, MICCAI 2010, Part I, LNCS 6361, 2010: 526-533.

[17] Czerwinski R N, Jones D L. Line and boundary detection in speckle images, IEEE Trans. Image Processing 1998;7: 1700-1714.

[18] Xiao C, Su Z, Chen Y. A Diffusion stick method for speckle suppression in ultrasonic images, Pattern Recognition Letters 2004; (25) $16: 1867-1877$

[19] González R C, Woods U. Digital image processing. Prentice Hall (2008).

[20] Perona P, Malik J. Scale space and edge detection using anisotropic Diffusion. IEEE Transactions on Pattern Analysis and Machine Intelligence, 1990, 12(7): 629-639.

[21] Black M J, Sapiro G. Marimont D H, Heeger D. Robust anisotropic Diffusion[J]. IEEE Transactions on Image Processing, 1998, 7(3): 412-432.

[22] Yezzi A. Modified Curvature Motion for Image Smoothing and Enhancement, IEEE Transaction on Medical Imaging, 1998, 7 (3).

[23] Qian Y, Zhang Y, Morgan M. A comparison of medical image segmentation methods for cerebral aneurysm computational hemodynamics. Biomedical Engineering and Informatics (BMEI), 2011 4th International Conference, 2011, 2: 901-904

[24] Brassel M, Bretin E. A modified phase field approximation for mean curvature flow with conservation of the volume, Mathematical Methods in the Applied Sciences 2011, 34(10): 1157-1180,

[25] Ibáñez L. The ITK Software Guide -Second Edition November 21,2005 .

[26] Boskamp T, Rinck D, Link, F, K " ummerlen B, Stamm G, Mildenberger P.. New vessel analysis tool for morphometric quantification and visualization of vessels in ct and $\mathrm{mr}$ imaging data sets. Radiographics, 2004 24(1) :287-297.

[27] Adams R and Bischof L, Seeded region growing, IEEE Trans. Pattern Anal. Machine Intell. 1994, 16: 641-647.

[28] Fan J, Yau DY, Elmagarmid AK, Aref WG. Automatic image segmentation by integrating color-edge extraction and seeded region growing. IEEE Trans Image Process. 2001;10(10):1454-66.

[29] Vanhoenacker PK, Heijenbrok-Kal MH,Van Heste R, Decramer I, Van Hoe L R, Wijins W, et al. Diagnostic performance of multidetector CT angiography for assessment of coronary artery disease: metaanalysis. Radiology $2007 ; 244: 419-428$.

[30] Miller JM, Rochitte CE, Dewey M, Arabab-Zadeh A, Ninuma H, Gottlieb I, et al. Diagnostic performance of coronary angiography by 64-row CT . N Engl J Med 2008 ; 359 : 2324 - 2336.

[31] Sun Z, Jiang W . Diagnostic value of multislice computed tomography angiography in coronary artery disease: a meta-analysis. Eur J Radiol $2006 ; 60: 279-286$.

[32] Hausleiter J, Meyer T, Hadamitzky M, Zankl M, Gerein P, Dorrler K, Kastrati A, et al. Non-invasive coronary computed tomographic angiography for patients with suspected coronary artery disease: the Coronary Angiography by Computed Tomography with the Use of a Submillimeter resolution (CACTUS) trial . Eur Heart J 2007 ; 28 : $3034-3041$.

[33] Budoff MJ, Dowe D, Jollis JG, Gitter M, Sutherland J, Halamert E, et al. Diagnostic performance of 64-multidetector row coronary computed tomographic angiography for evaluation of coronary artery stenosis in individuals without known coronary artery disease: results from the prospective multicenter ACCURACY (Assessment by Coronary Computed Tomographic Angiography of Individuals Undergoing Invasive Coronary Angiography) trial . J Am Coll Cardiol $2008 ; 52: 1724-1732$.

[34] Mowatt G, Cook JA, Hillis GS, Walker S, Fraser C, Jia X, et al 64-slice computed tomography angiography in the diagnosis and assessment of coronary artery disease: systematic review and metaanalysis. Heart 2008 ; 94 : 1386 - 1393

[35] Juergens KU, Fischbach R . Left ventricular function studied with MDCT . Eur Radiol 2006; $16: 342-357$.

[36] Mahnken AH, Koos R, Katoh M, Wildberger J E, Spuentrup E, Buecker A, et al. Assessment of myocardial viability in reperfused acute myocardial infarction using 16-slice computed tomography in comparison to magnetic resonance imaging . J Am Coll Cardiol 2005; $45: 2042-2047$

[37] Gerber BL, Belge B, Legros GJ, Lim P, Poncelet A, Pasquet A, et al. Characterization of acute and chronic myocardial infarcts by multidetector computed tomography: comparison with contrastenhanced magnetic resonance . Circulation $2006 ; 113: 823-$ 833.

[38] Lardo AC, Cordeiro MA, Silva C, Amado L C, George R T, Slaiaris A $\mathrm{P}$, et al . Contrast-enhanced multidetector computed tomography viability imaging after myocardial infarction: characterization of myocyte death, microvascular obstruction, and chronic scar. Circulation $2006 ; 113: 394-404$.

[39] Teshima H, Aoyagi S, Hayashida N, Shojima T, Takagi K, Arinaga K, et al. Dysfunction of an ATS valve in the aortic position: the first reported case caused by pannus formation. J Artif Organs $2005 ; 8$ : $270-273$.

[40] Teshima H, Hayashida N, Fukunaga S, Tayama E, Kawara T, Aoyagi $\mathrm{N}$, et al . Usefulness of a multidetector-row computed tomography scanner for detecting pannus formation. Ann Thorac Surg $2004 ; 77$ : $523-526$

[41] Teshima H, Hayashida N, Enomoto N, Aoyagi S, Okuda K, Uchida M . Detection of pannus by multidetector-row computed tomography. Ann Thorac Surg 2003; $75: 1631-1633$.

[42] Aoyagi S, Fukunaga S, Arinaga K, Shojima T, Ueda T . Prosthetic valve obstruction: diagnostic usefulness of cineradiography and multidetector-row computed tomography. Thorac Cardiovasc Surg $2007 ; 55: 517-519$

[43] Kim RJ, Weinsaft JW, Callister TQ, Min JK . Evaluation of prosthetic valve endocarditis by 64-row multidetector computed tomography Int J Cardiol 2007; 120 : e27 - e29 . Epub 2007 Jun 27. PubMed PMID: 17597237

[44] Tsai IC, Lin YK, Chang Y, Fu YC, Wang CC, Hsieh SR, et al . Correctness of multi-detector-row computed tomography for diagnosing mechanical prosthetic heart valve disorders using operative findings as a gold standard. Eur Radiol $2009 ; 19$ : $857-$ 867.

[45] Konen E, Goitein O, Feinberg MS, Eshet Y, Raanani E, Rimon U, et al. The role of ECG-gated MDCT in the evaluation of aortic and mitral mechanical valves: initial experience. AJR Am J Roentgenol $2008 ; 191: 26-31$.

[46] Ruggieri VG, Flecher E, Anselmi A, Lelong B, Corbineau H, Verhoye JPh et al. Long-Term Results of the Carpentier-Edwards Supraannular Aortic Valve Prosthesis. Ann Thorac Surg. 2012 Jul 7. 